\title{
Differential Impact of Resistance-Associated Mutations to Protease Inhibitors and Nonnucleoside Reverse Transcriptase Inhibitors on HIV-1 Replication Capacity
}

\author{
Szu-Min Hsieh,, ${ }^{1, *}$ Sung-Ching Pan,, ${ }^{1, *}$ Sui-Yuan Chang, ${ }^{2}$ Chien-Ching Hung, Wang-Huei Sheng, \\ Mao-Yuan Chen, and Shan-Chwen Chang ${ }^{1}$
}

\begin{abstract}
The effects of drug resistance on HIV-1 replication capacity have been studied, but data from clinical isolates are few. We accessed the patients with HIV-1 infection at the National Taiwan University Hospital who experienced virological failure. Genotypic susceptibility and replication capacity of clinical HIV-1 isolates were measured. There were 80 patients enrolled between September 2007 and August 2010. The HIV-1 replication capacity declined significantly with the increasing number of major resistance-associated mutations (RAMs) to protease inhibitors (PIs) $(p<0.001)$; however, it did not decline significantly with the increasing RAMs to first-line nonnucleoside analogue reverse transcriptase inhibitors (NNRTIs) $(p=0.098)$. Regarding the effects of resistance to antiretroviral drugs in salvage therapy, decreased replication capacity was noted with the increasing RAMs to darunavir/ritonavir $(p<0.001)$ and specific RAMs (L100I, K101P, and Y181C/I/V) to etravirine $(p<0.001)$. Although NNRTI-related RAMs have less remarkable effects, both PI- and NNRTI-related RAMs reduced replication capacity, especially RAMs to darunavir/ritonavir and etravirine, which are commonly used in salvage therapy for treatment of patients infected with highly resistant HIV. Thus, decreased viral fitness during the emergence of RAMs suggests the importance of continued optimal antiretroviral treatment even when virological failure was noted.
\end{abstract}

\section{Introduction}

$\mathbf{V}$ IROLOGICAL FAILURE OF antiretroviral therapy may be due to transmitted drug resistance or emergence of resistance during therapy. Transmission and acquisition of drug-resistant HIV have been documented as $6 \%$ to $16 \%$ resistance to at least one drug and 3\% to 5\% reduced susceptibility for more than one class of medication in the United States and Europe; thus, patients may face limited treatment options. ${ }^{1-3}$ However, clinical studies have suggested that development of drug resistance may not be associated with more rapid disease progression. ${ }^{4,5}$ Furthermore, treatment interruption in patients who fail to respond to antiretroviral therapy may reverse multidrug-resistant HIV-1 to wild-type virus and may thus be associated with a higher risk of disease progression or death than continued incompletely suppressive therapy. ${ }^{6}$

The benefit of persistent viral suppression despite the use of optimal therapy may be mediated by reductions in viral fit- ness associated with resistance-associated mutations (RAMs). When HIV develops resistance to antiretroviral therapy through the acquisition of mutations, reduced replication capacity and reduced pathogenicity may result. ${ }^{7,8}$ The impaired viral fitness associated with acquisition of drug resistance presumably results from alterations in natural substrate binding and catalytic activity that occur as a consequence of structural changes in reverse transcriptase, protease enzymes, or integrase..$^{9-11}$ Thus, if completely suppressive regimens are not available, continued incompletely suppressive therapy may theoretically provide benefits.

Studies regarding the effect of RAMs on different classes of antiretroviral drugs and HIV-1 replication capacity have been limited, and most data were produced by in vitro experiments on recombinant viruses from site-directed mutagenesis. ${ }^{12,13}$ We are specifically interested in darunavir/ritonavir (DRV/r) and etravirine (ETB) since they are essential components of salvage antiretroviral therapy for patients infected with

\footnotetext{
${ }^{1}$ Department of Internal Medicine, National Taiwan University Hospital and National Taiwan University College of Medicine, Taipei, Taiwan.

${ }^{2}$ Department of Laboratory Medicine, National Taiwan University Hospital, Taipei, Taiwan.

*These authors contributed equally to this work.
} 
drug-resistant HIV-1 in Taiwan ${ }^{14}$; thus, HIV-1 resistance to $\mathrm{DRV} / \mathrm{r}$ or ETR is a critical issue for patients who fail to respond to first-line antiretroviral therapy. In this study, we explored the effect of protease inhibitor (PI), including DRV /r, -related and/or nonnucleoside analogue reverse transcriptase inhibitor (NNRTI), including ETR-related RAMs on HIV-1 replication capacity by correlating the numbers of RAMs and susceptibility from genotypic resistance tests with the replication capacity of clinical HIV-1 isolates.

\section{Materials and Methods}

\section{Subjects}

At the National Taiwan University Hospital, a major referral center for HIV/AIDS in Taiwan, HIV-1-infected patients who experienced virological failure were tested for genotypic resistance to HIV-1 from September 2007 to August $2010 .{ }^{15}$ Virological failure was defined as a confirmed HIV RNA level $>400$ copies $/ \mathrm{ml}$ after 24 weeks of antiretroviral treatment, $>50$ copies $/ \mathrm{ml}$ after 48 weeks, or a repeated detectable HIV RNA level after prior suppression of viremia. ${ }^{1}$ Resistance testing was performed while the patients were receiving antiretroviral treatment or immediately ( $<4$ weeks) after discontinuation of the failed regimen. This study was approved by the institutional review board of the hospital, and informed consent was obtained from all subjects before analysis. The human experimentation guidelines of the U.S. Department of Health and Human Services were followed in the conduct of clinical research.

\section{Genotypic resistance assay}

The genotypic resistance assay has been described previously. ${ }^{15}$ Briefly, total RNA was extracted from plasma using the QIAamp Viral RNA Mini Kit (Qiagen, Valencia, CA) according to the manufacturer's protocol. Polymerase chain reaction was performed in a final volume of $50 \mu \mathrm{l}$ containing $20 \mathrm{mmol} /$ liter Tris- $\mathrm{HCl}$ (pH 8.4), $50 \mathrm{mmol} /$ liter $\mathrm{KCl}$, $1.5 \mathrm{mmol} /$ liter $\mathrm{MgCl}_{2}, 0.2 \mathrm{mmol} /$ liter of each deoxynucleoside triphosphate, $0.2 \mu \mathrm{mol} /$ liter of each specific primer, and $2.5 \mathrm{U}$ of platinum Taq DNA polymerase (Invitrogen Life Technologies, Grand Island, NY). Population-based nucleotide sequence analysis of the polymerase chain reaction fragments was conducted using an automatic sequencer (3100 Avent Genetic Analyzer; Applied Biosystems, Foster City, CA).

\section{Genotypic resistance levels}

Genotypic resistance levels were assessed by determining the number of RAMs. The following major mutations associated with resistance to currently available PIs were detected: V32I, M46I/L, I47V / A, I50L/V, I54M/L, L76V, V82A/F/L/ $\mathrm{S} / \mathrm{T}, \mathrm{I} 84 \mathrm{~V}, \mathrm{~N} 88 \mathrm{~S}$, and L90M. The following major mutations associated with resistance specifically to $\mathrm{DRV} / \mathrm{r}$ were detected: I47V, I50V, I54M/L, L76V, and I84V. The following mutations associated with resistance to first-line NNRTIs (nevirapine and efavirenz) were detected: L100I, K101P, K103N/S, V106A/M, V108I, Y181C/I, Y188C/L/H, G190S/ $\mathrm{A}$, and $\mathrm{P} 225 \mathrm{H}$. The following mutations associated with resistance specifically to ETR were detected: V90I, A98G, L100I, K101E/H/P,V106I,E138A/G/K/Q,V179D/F/T, Y181C/I/V, G190S/A, and M230L. The categories of drug susceptibility (susceptible, potential low-level resistant, low-level resistant, intermediate-level resistant, and high-level resistant) were classified according to the definition of the Stanford University HIV Drug Resistance Database Program (http:// hivdb.stanford.edu/index.html).

\section{Estimation for HIV-1 viral fitness: comparative assay for replication capacity}

The comparative replication assay to estimate HIV-1 viral fitness was described previously and has been modified here. $^{16,17} \mathrm{~A}$ total of $10^{6} \mathrm{CD} 8$-depleted peripheral blood mononuclear cells (PBMCs) from 10 naive HIV-1-infected subjects were cocultured with the same number of CD8depleted PBMCs from HIV-1-negative donors at $37^{\circ} \mathrm{C}$ and $5 \%$ $\mathrm{CO}_{2}$ in a humidified chamber, and cellular proviral HIV-1 DNA was measured using the Quanti-Kin Detection System, HIV Total DNA Quantity (Symbiosis, Asti, Italy). Capsid protein (p24 antigen) production was measured in culture supernatants on day 7 using an enzyme immunoassay kit (Beckman Coulter, Fullerton, CA). A log-linear plot was constructed using the p24 antigen levels on day 7 (on the $y$-axis) versus baseline cellular proviral HIV-1 DNA (on the $x$-axis). The log-linear plot was used as a reference curve. A total of $10^{6}$ CD8-depleted PBMCs derived from the patients with virological failure to antiretroviral drugs were cocultured with the same number of CD8-depleted PBMCs from HIV-1-negative donors. The replication capacity of HIV-1 isolates from the subjects was calculated as the ratio multiplied by 100 of the p24 antigen level of isolates (on day 7) over the p24 antigen level determined on the reference curve according to the baseline cellular proviral HIV-1 DNA levels. All assays were performed in triplicate. In our preliminary experiences, a plasma HIV-1 RNA level greater than 3,000 copies $/ \mathrm{ml}$ and a CD4 ${ }^{+} \mathrm{T}$ cell count greater than $100 / \mu \mathrm{l}$ in peripheral blood were required to perform the replication assay; otherwise, the results from the assay would be highly variable and thus unreliable. Thus, the subjects who met the criteria of virological failure were enrolled only if their CD4 ${ }^{+}$ T cell count exceeded $100 / \mu \mathrm{l}$ and their plasma HIV-1 RNA level exceeded 3,000 copies / $\mathrm{ml}$.

\section{Statistical analysis}

Statistical analysis was performed using SPSS statistical software, version 6.1.3 (SPSS Inc., Chicago, IL). For analysis of continuous data between two groups, the nonparametric Mann-Whitney $U$-test was used. One-way analysis of variance (ANOVA) was used if $\geq 3$ groups were compared. $p<0.05$ was considered significant.

\section{Results \\ Characteristics of subjects}

Between September 2007 and August 2010, 80 subjects with HIV-1 were enrolled and analyzed. Characteristics of the subjects are listed in Table 1 . Of note, all subjects were homosexual men. Age, $\mathrm{CD}^{+}{ }^{+} \mathrm{T}$ cell count, and plasma HIV RNA level at baseline or at enrollment, as well as duration of exposure to antiretroviral therapy, were similar between the enrolled subjects exposed to PI-based regimens and those exposed to NNRTI-based regimens. 
Table 1. Selected Characteristics of Enrolled Subjects

\begin{tabular}{|c|c|c|c|}
\hline & $\begin{array}{l}\text { Exposed to PI-based } \\
\text { regimens }(\mathrm{n}=46)^{\mathrm{a}}\end{array}$ & $\begin{array}{l}\text { Exposed to NNRTI-based } \\
\text { regimens }(\mathrm{n}=52)^{\mathrm{a}}\end{array}$ & $\mathrm{p}$ value \\
\hline $\operatorname{Sex}(\mathrm{M} / \mathrm{F})$ & $46 / 0$ & $52 / 0$ & \\
\hline Age $($ mean $\pm S D)$ & $33.7 \pm 8.8$ & $35.1 \pm 7.6$ & 0.4 \\
\hline Baseline CD4 ${ }^{+}$cell count $(/ \mu \mathrm{l})($ mean $\pm \mathrm{SD})$ & $245.1 \pm 90.7$ & $256.8 \pm 85.6$ & 0.513 \\
\hline Baseline plasma HIV-1 DNA $\left(\times 10^{3}\right.$ copies $\left./ \mathrm{ml}\right)($ mean \pm SD) & $28 \pm 19$ & $31 \pm 17$ & 0.411 \\
\hline $\mathrm{CD}^{+}$cell count at enrollment $(/ \mu \mathrm{l})($ mean $\pm \mathrm{SD})$ & $284.5 \pm 86.7$ & $267.8 \pm 78.5$ & 0.319 \\
\hline Plasma HIV-1 DNA at enrollment $\left(\times 10^{3}\right.$ copies $\left./ \mathrm{ml}\right)($ mean $\pm S D)$ & $19 \pm 12$ & $16 \pm 13$ & 0.24 \\
\hline Duration of exposure to antiretroviral drugs (weeks) (mean $\pm S D$ ) & $74 \pm 21$ & $68 \pm 23$ & 0.183 \\
\hline
\end{tabular}

${ }^{a}$ A total of 18 subjects were exposed to both PI-based and NNRTI-based regimens.

PI, protease inhibitors; NNRTI, nonnucleoside analogue reverse transcriptase inhibitors.

\section{Effects of number of RAMs on replication capacity}

To assess the effect of the major RAMs to PIs on HIV-1 fitness, we determined the association between the number of major RAMs to PIs and the replication capacity of HIV-1. We found that the HIV-1 replication capacity declined significantly with the increasing number of major RAMs to PIs $(p<0.001$ by one-way ANOVA, Fig. 1a). The replication capacity decreased to $<50 \%$ (mean, $49.8 \%$ ) when the number of major RAMs to PIs was $\geq 4$. However, the mean HIV-1 replication capacity remained $>60 \%$ regardless of the number of RAMs to first-line NNRTIs and did not decline significantly with the increasing number of RAMs to first-line NNRTIs $(p=0.098$ by one-way ANOVA, Fig. $1 \mathrm{~b})$.

To determine the cross-effects of RAMs to PIs and RAMs to NNRTIs on HIV-1 fitness, we assessed the distribution of HIV-1 replication capacity according to the number of major RAMs to PIs and RAMs to NNRTIs in a triaxial figure (Fig. 1c). We found that the replication capacity declined markedly with the increasing number of major RAMs to PIs if there were the same number of RAMs to NNRTIs, and the decline in replication capacity was less remarkable with the increasing number of RAMs to NNRTIs if there were the same number of major RAMs to PIs.

\section{Effects of HIV-1 resistance to DRV/r on replication capacity}

To determine the effect of the major RAMs to DRV/r on HIV-1 fitness, we assessed the association between the number of major RAMs to DRV/r and the replication capacity of HIV-1. No HIV-1 isolates from the enrolled subjects had $\geq 4$ major RAMs to DRV / r among I47V , I50V, I54M/L, L76V, and I84V. We found that the HIV-1 replication capacity declined significantly with the increasing number (from 0 to 3 ) of major RAMs to DRV / r $(p<0.001$ by one-way ANOVA, Fig. 2a). The replication capacity was also compared between HIV-1 with major RAMs to DRV/r and HIV-1 with major RAMs to PIs but not to DRV/r (i.e., V32I, M46I/L, I47A, V82A/F/L/S/T, $\mathrm{N} 88 \mathrm{~S}$, and L90M) to determine their relative effect; the replication capacity (mean \pm SD) was as follows: one mutation, $56.5 \% \pm 10.7 \%$ versus $74.2 \% \pm 12.3 \%, p<0.001$; two mutations, $38.3 \% \pm 13.6 \%$ versus $60.6 \% \pm 11.5 \%, p<0.001$; and three mutations, $23.0 \% \pm 7.8 \%$ versus $49.7 \% \pm 10.8 \%, p=0.012$, respectively. The results showed that with the same number of major RAMs, the major RAMs to DRV/r had an even more remarkable effect on replication capacity than the major RAMs to PIs but not to DRV/r.
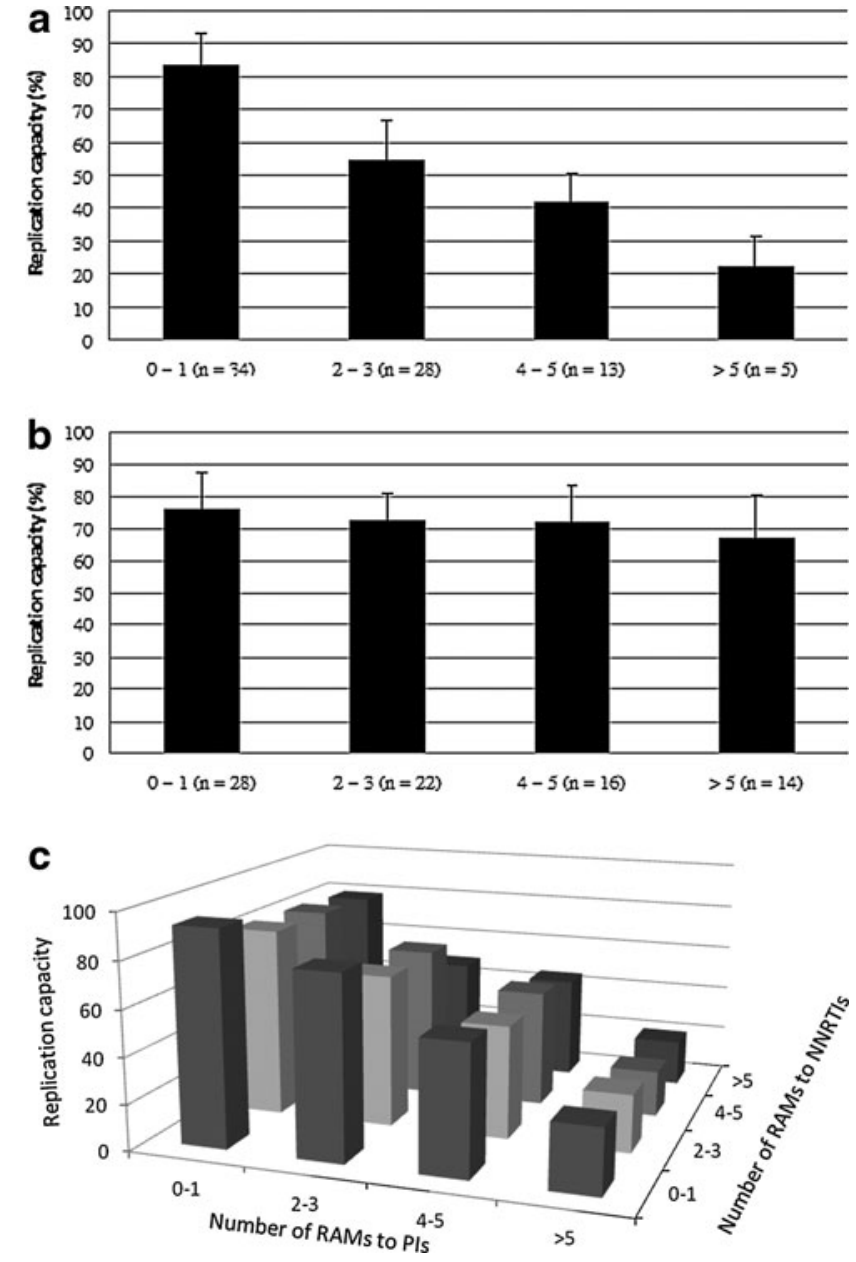

FIG. 1. The effect of resistance to antiretroviral drugs on replication capacity. (a) Association between the number of major resistance-associated mutations (RAMs) to protease inhibitors (PIs) and replication capacity. The major RAMs to PIs include V32I, M46IL, I47VA, I50LV, I54ML, L76V, V82AFLST, I84V, N88S, and L90M $[p<0.001$ by one-way analysis of variance (ANOVA)]. (b) Association between the number of RAMs to first-line nonnucleoside analogue reverse transcriptase inhibitors (NNRTIs; nevirapine and efavirenz) and replication capacity. The mutations associated with resistance to first-line NNRTIs included L100I, K101P, K103NS, V106AM, V108I, Y181CI, Y188CLH, G190SA, and P225H ( $p=0.098$ by one-way ANOVA). (c) Association between the number of RAMs to PIs, the number of RAMs to NNRTIs, and replication capacity. 

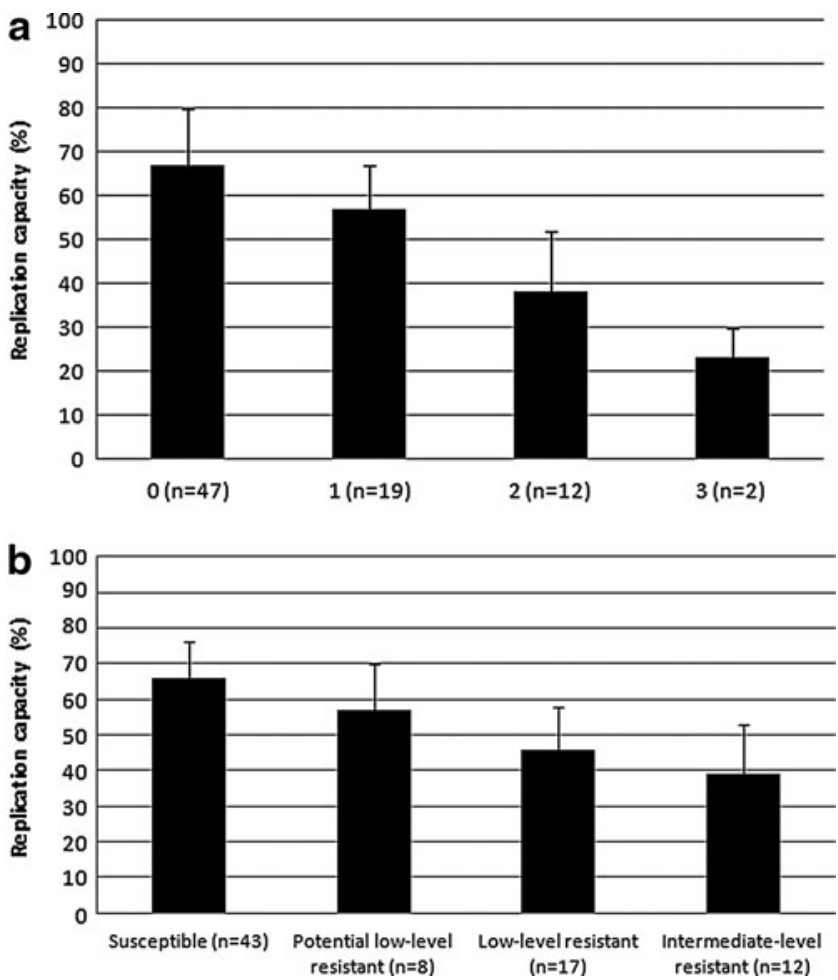

FIG. 2. The effect of resistance to darunavir/ritonavir on replication capacity. (a) Association between the number of resistance-associated mutations (RAMs) to darunavir/ritonavir $(\mathrm{DRV} / \mathrm{r}$ ) and replication capacity. The major RAMs to DRV/r included I47V, I50V, I54ML, L76V, and I84V [ $p<0.001$ by oneway analysis of variance (ANOVA)]. (b) Association between HIV-1 susceptibility to $\mathrm{DRV} / \mathrm{r}$ and replication capacity $(p<0.001$ by one-way ANOVA).
We also assessed the drug susceptibility to DRV/r. No HIV-1 isolate from the enrolled subjects was categorized as high-level resistance. We found that the HIV-1 replication capacity declined significantly with decreasing susceptibility to DRV/r $(p<0.001$ by one-way ANOVA, Fig. $2 \mathrm{~b})$.

\section{Effects of HIV-1 resistance to ETR on replication capacity}

To determine the effect of the RAMs to ETR on HIV-1 fitness, we assessed the association of the number of RAMs to ETR and the replication capacity of HIV-1. We found that the mean HIV-1 replication capacity remained $>65 \%$ regardless of the number of RAMs to ETR and did not decline significantly with the increasing number of RAMs ( $p=0.081$ by one-way ANOVA, Fig. 3a). However, we found that the HIV-1 replication capacity declined significantly with decreasing susceptibility to ETR ( $p<0.001$ by one-way ANOVA, Fig. 3b).

To explain the discrepancy, we assessed the effect of selected RAMs that were considered to have higher relative weights on susceptibility and clinical response, including L100I, K101P, and $\mathrm{Y} 181 \mathrm{C} / \mathrm{I} / \mathrm{V},{ }^{18}$ on replication capacity. No HIV-1 isolates from the enrolled subjects had more than two mutations among these selected RAMs. We found that the HIV-1 replication capacity declined significantly with the increasing number (from 0 to 2 ) of these selected RAMs to ETR $(p<0.001$ by one-way ANOVA, Fig. 3c).

\section{Discussion}

In this study, we analyzed the replication capacity of clinical HIV-1 isolates from 80 treatment-experienced subjects. Our data showed that the accumulation of drug resistance could have an effect on replication capacity of clinical HIV-1 isolates. Generally, the RAMs to PIs have a more remarkable $\mathbf{a}_{100}$

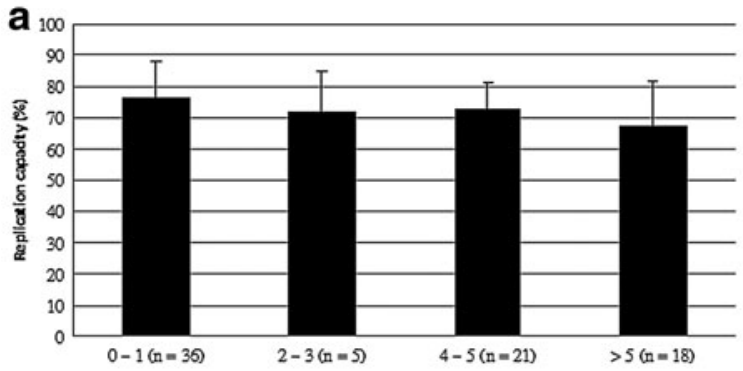

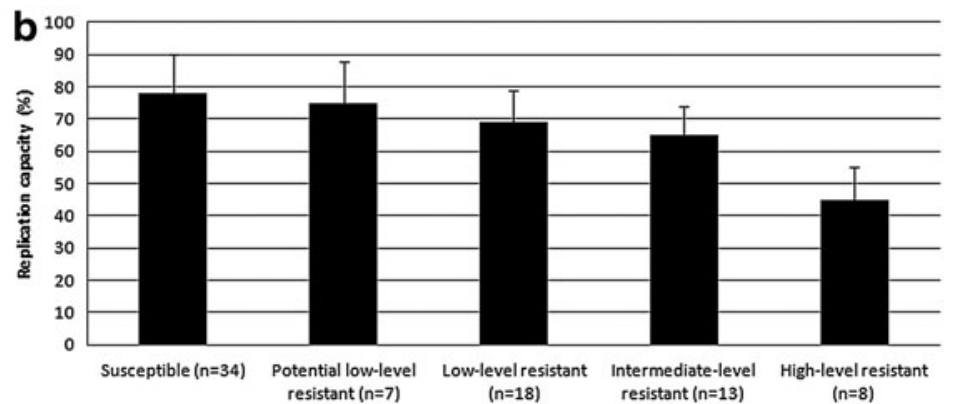

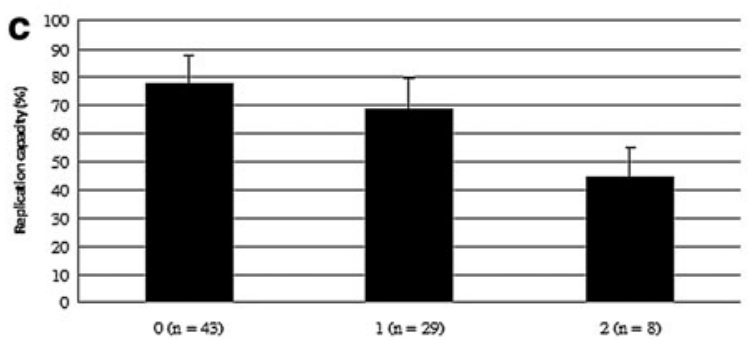

FIG. 3. The effect of resistance to etravirine on replication capacity. (a) Association between the number of resistanceassociated mutations (RAMs) to etravirine (ETR) and replication capacity. The mutations associated with resistance to ETR include V90I, A98G, L100I, K101EHP, V106I, E138AGKQ, V179DFT, Y181CIV, G190SA, and M230L [ $p=0.081$ by one-way analysis of variance (ANOVA)]. (b) Association between HIV-1 susceptibility to ETR and replication capacity $(p<0.001$ by one-way ANOVA). (c) Association between the number of selected RAMs (including L100I, K101P, Y181C/I/V) to ETR and replication capacity $(p<0.001$ by one-way ANOVA). 
effect on decreasing replication capacity than RAMs to NNRTIs; reduced replication capacity can also be noted with the increased RAMs to relatively newly introduced antiretroviral drugs such as DRV/r and ETR.

HIV viral fitness, which refers to the ability of a virus to reproduce within a host, was mimicked in vitro by replication capacity. Because replication of HIV within a host is a complicated task involving target cell entry, reverse transcription, integration, gene expression, assembly, egress, maturation, and control of the host cell for optimal replication and virion production, ${ }^{19}$ the function of the virus could be easily influenced because of mutation, led by the error-prone process of replication and pressure from antiretroviral therapy. Viral fitness, as replication capacity, can lead to further correlation with clinical outcome, and it has gained attention with increasing drug resistance among patients experienced with antiretroviral therapy or naive patients with transmitted resistance., ${ }^{2,3}$

A number of methods have been developed to measure viral fitness, including replication kinetics and competitive culture assays, but they are time consuming and labor intensive. ${ }^{20}$ Furthermore, prior studies on the effect of drug resistance on HIV-1 replication capacity were limited to recombinant viruses from site-directed mutagenesis. ${ }^{12,13}$ However, the HIV replication process intensively involves the interaction between the host and virus, so the replication capacity through recombinant virus may not properly reflect the behavior of clinical isolates with a complicated resistance pattern and the effect of background polymorphisms of the virus within the host. Our modified simple method may provide a clinically practical measurement for replication capacity of clinical isolates.

A prior study with a small number of cases $(N=28)$ involving the replication capacity of HIV-1 strains from patients noted that the RAMs to NNRTIs had a minimal effect on replication capacity of HIV-1 when compared with the RAMs to PIs. ${ }^{21}$ Our study further supported this finding because the dose-response was observed between accumulated RAMs and PIs to decreased replication and the dose-related phenomenon was less markedly demonstrated in RAMs to NNRTIs. Furthermore, the triaxial figure (Fig. 1c) shows that while combining the effect of RAMs to PIs and RAMs to NNRTIs, the effect of RAMs to PIs predominated on HIV-1 replication capacity. This can be partially explained by the fact that the PIs act on the active site of the enzyme, whereas the active site of NNRTIs is more distant. The more the mutation occurred within the active site of the enzyme, the more the enzymatic activity can be affected and replication capacity could decrease. ${ }^{22}$

As to the correlation between replication capacity and treatment outcome, the ARGENTA trial $(N=139)$ in 19992000 revealed that after adjusting baseline CD4 cell counts, HIV-1 RNA levels, and phenotypic susceptibility to the rescue regimen, higher viral replication capacity may predict worse treatment outcome. ${ }^{13}$ Thus, because the drug resistance to PIs occurred with decreased replication capacity, clinical benefit would be expected by keeping selective antiretroviral drug pressure despite drug resistance. On the other hand, for the novel NNRTI such as ETR, the specific RAMs to ETR led to decreased replication capacity in our study. Whether this finding supports the use of ETR rather than other first-line NNRTIs for patients with NNRTI resistance warrants further clinical studies.
The study has several limitations. Even though our data revealed that replication capacity decreases with increased number of RAMs to PIs, including DRV/r, we do not know the possible differential effect of different RAMs. In the study on the effect of raltegravir resistance by $\mathrm{Hu}$ and Kuritzkes, the virus with a Q148H mutation was less fit than the virus with an $\mathrm{N} 155 \mathrm{H}$ mutation and the wild-type virus; however, the presence of the G140S, E92Q, and E138K mutation partially restored the replication capacity. ${ }^{12}$ However, as compared to site-directed mutagenesis and study in recombinant virus, our study can better demonstrate the issue of clinical isolates in a clinical setting. The effect of specific RAMs could be studied by further observation with larger sample sizes.

Selection bias may exist because the cases were derived from only one major medical center rather than from a nationwide survey. Baseline resistance information is lacking, so we do not know how many major mutations developed during antiretroviral treatment with PI-containing regimens. Furthermore, the effect of medication adherence on the emergence of resistance, which may bias the interpretation of the results, could not be assessed in this study.

The external validity of this study is limited because all 80 subjects were men with a risk factor of homosexual behavior. However, viral biology may hardly be affected by human behavior, and we expect that the results can be applied to other populations, although further studies are warranted.

In conclusion, the increasing resistance to PIs or NNRTIs implies that the progressive accumulation of RAMs may impede the effectiveness of antiretroviral therapy but also decrease viral fitness. These data suggest the importance of continued optimal antiretroviral treatment even when the viral load cannot be reduced to an undetectable level because of resistance.

\section{Acknowledgments}

We are indebted to the Centers for Diseases Control, Taiwan for providing financial support for this study $(\mathrm{DOH}-$ 96-DC-1009). S.M. Hsieh also received a grant from the Department of Health, Taiwan (DOH100-TD-B-111-001). The funding organizations were not involved in designing or conducting the study, the data collection, management, analysis, and interpretation, or the preparation and approval of the manuscript.

S.M. Hsieh, C.C. Hung, and S.C. Chang initiated the study. S.M. Hsieh, C.C. Hung, W.H. Sheng, and M.Y. Chen enrolled the patients. S.M. Hsieh and S.Y. Chang performed the laboratory work. S.M. Hsieh and S.C. Pan prepared the article.

\section{Author Disclosure Statement}

No competing financial interests exist.

\section{References}

1. Panel on Antiretroviral Guidelines for Adults and Adolescents: Guidelines for the Use of Antiretroviral Agents in HIV1-Infected Adults and Adolescents. Department of Health and Human Services. Available at http://aidsinfo.nih.gov/ contentfiles/lvguidelines/adultandadolescentgl.pdf. Accessed August 3, 2012. 
2. Weinstock HS, Zaidi I, Heneine W, et al.: The epidemiology of antiretroviral drug resistance among drug-naive HIV-1infected persons in 10 US cities. J Infect Dis 2004;189: 2174-2180.

3. Wensing AMJ, van de Vijver DA, Angarano G, et al.: Prevalence of drug-resistant HIV-1 variants in untreated individuals in Europe: Implications for clinical management. J Infect Dis 2005;192:958-966.

4. Lucas GM, Gallant JE, and Moore RD: Relationship between drug resistance and HIV-1 disease progression or death in patients undergoing resistance testing. AIDS 2004;18:1539 1548.

5. Gange SJ, Schneider MF, Grant RM, et al.: Genotypic resistance and immunologic outcomes among HIV-1-infected women with viral failure. J Acquir Immune Defic Syndr 2006;41:68-74.

6. Lawrence J, Mayers DL, Hullsiek KH, et al.: Structured treatment interruption in patients with multidrug-resistant human immunodeficiency virus. N Engl J Med 2003;49:837-846.

7. Deeks SG, Wrin T, Liegler T, et al.: Virologic and immunologic consequences of discontinuing combination antiretroviraldrug therapy in HIV-infected patients with detectable viremia. N Engl J Med 2001;344:472-480.

8. Ledergerber B, the PLATO Collaboration: Predictors of trend in CD4-positive T-cell count and mortality among HIV-1infected individuals with virological failure to all three antiretroviral-drug classes. Lancet 2004;364:51-62.

9. Dauber DS, Ziermann R, Parkin N, et al.: Altered substrate specificity of drug-resistant human immunodeficiency virus type 1 protease. J Virol 2002;76:1359-1368.

10. Malim $\mathrm{MH}$ and Emerman M: HIV-1 sequence variation: Drift, shift, and attenuation. Cell 2001;104:469-472.

11. Lee MC, Deng J, Briggs JM, et al.: Large-scale conformational dynamics of the HIV-1 integrase core domain and its catalytic loop mutants. Biophys J 2005;88:3133-3146.

12. $\mathrm{Hu} \mathrm{Z}$ and Kuritzkes DR: Effect of raltegravir resistance mutations in HIV-1 integrase on viral fitness. J Acquir Immune Defic Syndr 2010;55:148-155.

13. De Luca A, Weidler J, Giambenedetto SD, et al.: Association of HIV-1 replication capacity with treatment outcomes in patients with virologic treatment failure. J Acquir Immune Defic Syndr 2007;45:411-417.

14. Guideline on HIV Diagnosis and Treatment, 3rd ed. Center for Disease Control, Taipei, ROC, 2010.

15. Chang SY, Chen MY, Lee CN, et al.: Trends of antiretroviral drug resistance in treatment-naive patients with human immunodeficiency virus type 1 infection in Taiwan. J Antimicrob Chemother 2008;61:689-693.

16. Lu J and Kuritzkes DR: A novel recombinant marker virus assay for comparing the relative fitness of HIV-1 reverse transcriptase variants. J Acquir Immune Defic Syndr 2001;27:7-13.

17. $\mathrm{Hu} \mathrm{Z}$, Giguel F, Hatano $\mathrm{H}$, et al.: Fitness comparison of thymidine analog resistance pathways in human immunodeficiency virus type 1. J Virol 2006;80:7020-7027.

18. Johnson VA, Calvez V, Gunthard HF, et al.: 2011 update of the drug resistance mutations in HIV-1. Top Antivir Med 2011;19:156-164.

19. Greene WC and Peterlin BM: Charting HIV's remarkable voyage through the cell: Basic science as a passport to future therapy. Nat Med 2002;8:673-680.

20. Bates M, Wrin T, Huang W, et al.: Practical applications of viral fitness in clinical practice. Curr Opin Infect Dis 2003; 16:11-18.

21. Nicastri E, Sarmati L, d'Ettorre G, et al.: Replication capacity, biological phenotype, and drug resistance of HIV strains isolated from patients failing antiretroviral therapy. J Med Virol 2003;69:1-6.

22. Deeks SG: Non-nucleoside reverse transcriptase inhibitor resistance. J Acquir Immune Defic Syndr 2001;26:S25-S33.

Address correspondence to: Szu-Min Hsieh Department of Internal Medicine National Taiwan University Hospital and National Taiwan University College of Medicine

Taipei 100 Taiwan

E-mail: hsmaids@hotmail.com 